\title{
Multi-Wavelength Photobiomodulation Therapy Combined with Static Magnetic Field on Long-Term Pulmonary Complication after COVID-19: A Case Report
}

\author{
Shaiane Silva Tomazoni ${ }^{1, *}$, Douglas Scott Johnson ${ }^{2}$ and Ernesto Cesar Pinto Leal-Junior 1,3,4 \\ 1 Physiotherapy Research Group, Department of Global Public Health and Primary Care, University of Bergen, \\ 5020 Bergen, Norway; ernesto.leal.junior@uninove.br \\ 2 Multi Radiance Medical, Solon, OH 44139, USA; djohnson@multiradiance.com \\ 3 Laboratory of Phototherapy and Innovative Technologies in Health (LaPIT), Post-Graduate Program in \\ Rehabilitation Sciences, Nove de Julho University, São Paulo 01525-000, Brazil \\ 4 ELJ Consultancy, Avenida Chibarás, São Paulo 04076-000, Brazil \\ * Correspondence: Shaiane.Tomazoni@uib.no
}

Citation: Tomazoni, S.S.; Johnson, D.S.; Leal-Junior, E.C.P. Multi-Wavelength Photobiomodulation Therapy Combined with Static Magnetic Field on Long-Term Pulmonary Complication after COVID-19: A Case Report. Life 2021, 11, 1124. https://doi.org/10.3390/ life11111124

Academic Editors: Luisa Zupin and Sergio Crovella

Received: 7 October 2021

Accepted: 20 October 2021

Published: 22 October 2021

Publisher's Note: MDPI stays neutral with regard to jurisdictional claims in published maps and institutional affiliations.

Copyright: (c) 2021 by the authors. Licensee MDPI, Basel, Switzerland. This article is an open access article distributed under the terms and conditions of the Creative Commons Attribution (CC BY) license (https:// creativecommons.org/licenses/by/ $4.0 /)$.

\begin{abstract}
Introduction: Photobiomodulation therapy, alone (PBMT) or combined with a static magnetic field (PBMT-sMF), has been demonstrated to be effective in the regeneration of tissues, modulation of inflammatory processes, and improvement in functional capacity. However, the effects of PBMT-sMF on the pulmonary system and COVID-19 patients remain scarce. Therefore, in this case report, we demonstrated the use of PBMT-sMF for peripheral oxygen saturation, pulmonary function, massive lung damage, and fibrosis as a pulmonary complication after COVID-19. Case report: A 53-year-old Mexican man who presented with decreased peripheral oxygen saturation, massive lung damage, and fibrosis after COVID-19 received PBMT-sMF treatment once a day for 45 days. The treatment was irradiated at six sites in the lower thorax and upper abdominal cavity and two sites in the neck area. We observed that the patient was able to leave the oxygen support during the treatment, and increase his peripheral oxygen saturation. In addition, the patient showed improvements in pulmonary severity scores and radiological findings. Finally, the patient presented with normal respiratory mechanics parameters in the medium-term, indicating total pulmonary recovery. Conclusions: The use of PBMT-sMF may potentially lead to safe treatment of and recovery from pulmonary complications after COVID-19, with regard to the structural and functional aspects.
\end{abstract}

Keywords: low-level laser therapy; COVID-19; rehabilitation; pulmonary complication; case reports

\section{Introduction}

Severe acute respiratory syndrome coronavirus (SARS-CoV-2) is the causative agent of the novel coronavirus disease (COVID-19) [1]. The clinical features triggered by SARSCoV-2 infection may differ in each patient. Patients may be asymptomatic or, in some cases, patients may develop life-threatening sepsis [1,2]. In its early stages, SARS-CoV2 targets pneumocytes and nasal and bronchial epithelial cells, and it infects and kills $\mathrm{T}$ lymphocyte cells $[2,3]$. In later stages, there is an enhanced inflammatory response when the virus infects pulmonary capillary endothelial cells [4]. In addition, SARS-CoV-2 promotes endothelial barrier disruption, dysfunctional alveolar-capillary oxygen transmission, and impaired oxygen diffusion capacity [2]. These pulmonary characteristic features caused by SARS-CoV-2 may persist as pulmonary complications after treating COVID-19. There is evidence that a considerable proportion of patients have a pulmonary diffusion abnormality 6 months after COVID-19 symptoms onset [5].

Multi-wavelength photobiomodulation therapy (PBMT) combined with static magnetic field (PBMT-sMF) may be a non-pharmacological alternative intervention for the treatment of persistent pulmonary complications after COVID-19. PBMT is a light therapy that uses non-ionizing light sources such as low-level lasers, light emitting diodes, and 
broadband light from the visible to the infrared spectra to promote pain relief, regenerate tissues, and modulate inflammatory processes [6,7]. In the respiratory system, PBMT has been able to modulate pulmonary inflammation and relieve bronchial hyperresponsiveness in experimental models [8-10], in addition to improving the functional capacity of patients with chronic obstructive pulmonary disease [11]. In recent years, PBMT has been used in combination with a static magnetic field to generate greater electron transfer and, consequently, better effects on cell metabolism [12]. The positive effects of PBMT-sMF have been shown to be similar to those of PBMT alone, such as ergogenic effects, inflammatory process modulation, and pain relief [13-15]. However, currently, there is insufficient evidence regarding the effects of PBMT-sMF on the respiratory system. In addition, there is a lack of evidence on the effects of PBMT alone or PBMT-sMF on pulmonary complications after COVID-19. Here, we report the use of PBMT-sMF on peripheral oxygen saturation $\left(\mathrm{SpO}_{2}\right)$, pulmonary function, massive lung damage, and fibrosis as a pulmonary complication after COVID-19.

\section{Case Report}

A 53-year-old Mexican man, who was overweight and had a history of serological antibody tests for toxocariasis positive, brucellosis, gout, conjunctivitis, rhinitis, seasonal allergies, sneezing, and nasal congestion, presented with generalized pain, fatigue, intermittent fever $\left(38.5-40^{\circ} \mathrm{C}\right)$, dry cough, and dyspnea. SARS-CoV-2 diagnosis was confirmed by reverse transcription polymerase chain reaction (RT-PCR) using a nasopharyngeal swab. The patient was admitted to the hospital with an $\mathrm{SpO}_{2}$ of $74 \%$ and oxygen support of $14 \mathrm{~L} / \mathrm{min}$. Chest radiography demonstrated massive lung damage and fibrosis caused by severe pneumonia. At the hospital, the patient was treated with ceftriaxone and prednisone, along with enoxaparin injections and oral aspirin. After 10 days at the hospital, the patient improved, and he was discharged from the hospital. However, the patient still presented with generalized fatigue, and oxygen support $(2 \mathrm{~L} / \mathrm{min}$ ) was still required for sleeping and eating. Therefore, ten days after discharge from the hospital, the patient sought complementary treatment with PBMT-sMF to improve his health status.

\subsection{Pretreatment Clinical Findings}

The patient's response to the PBMT-sMF treatment was evaluated by monitoring $\mathrm{SpO}_{2}[16,17]$ from the time of admission to the hospital (baseline), until discharge from the hospital, 10 days after the start of treatment, immediately after the end of the treatment, and 4 months after the end of the treatment with PBMT-sMF. In addition, we evaluated the progression of the imaging findings in the chest $\mathrm{X}$-ray from the first $\mathrm{X}$-ray at baseline, until 10 days after the start of the treatment, and 4 months after the end of the treatment with PBMT-sMF. Finally, to quantify the extent of SARS-CoV-2 infection in the lungs, the severity score was calculated from chest $X$-rays. The adapted and simplified Radiographic Assessment of Lung Edema (RALE) score was used [18,19]. A score ranging from 0 to 4 was assigned to each lung based on the extent of consolidation or ground glass opacity: 0 , no involvement; $1,<25 \%$ involvement; $2,25-50 \%$ involvement; $3,50-75 \%$ involvement; and $4,>75 \%$ involvement. The final severity score was calculated as the sum of the scores for each lung. The RALE score was calculated at baseline, 10 days after the start of treatment, and 4 months after the end of the treatment with PBMT-sMF.

\subsection{Intervention}

Ten days after discharge from the hospital, the patient started the multi-wavelength PBMT-sMF treatment. PBMT-sMF was irradiated using a cordless, portable MR5 LaserShower ${ }^{\mathrm{TM}}$ device (Multi-Radiance Medical ${ }^{\mathrm{TM}}$, Solon, OH, USA). Six sites in the lower thorax/upper abdominal cavity were irradiated in addition to two sites in the neck area (Figure 1). At each treatment session, the patient received a total of $31.50 \mathrm{~J}$ per irradiated site. The treatment time per site was $60 \mathrm{~s}$, yielding $480 \mathrm{~s}$ per treatment session. The treatment 
was performed once daily for 45 days. The dose and irradiation sites were established based on previous studies $[11,20]$. Table 1 describes the PBMT-sMF parameters.

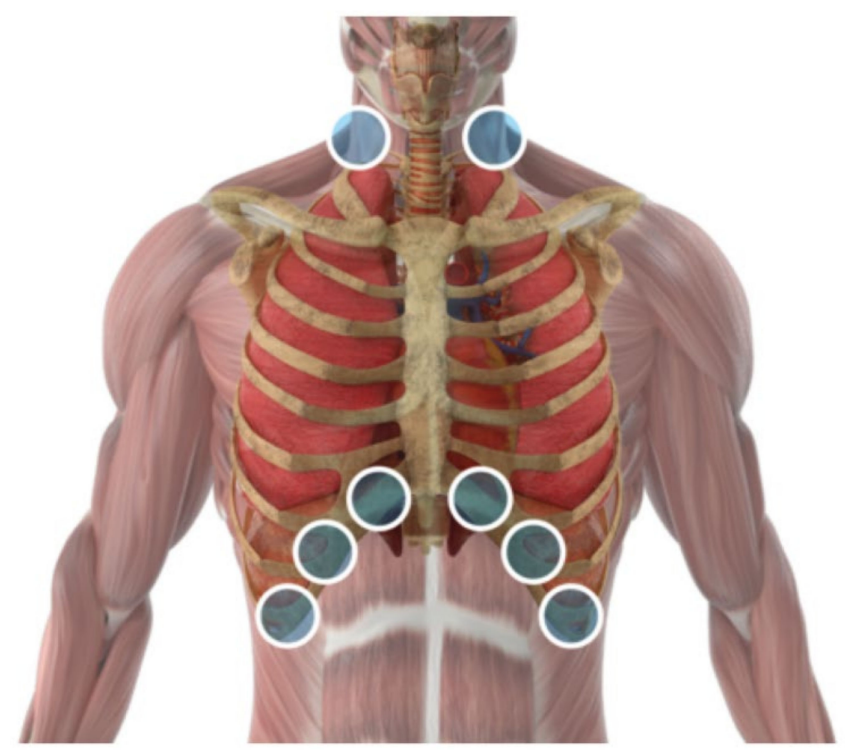

Figure 1. Intervention sites where PBMT-sMF was irradiated. Six sites in the lower thorax/upper abdominal cavity and two sites in the neck area.

Table 1. Full description of the PBMT-sMF parameters.

\begin{tabular}{|c|c|c|}
\hline & $\begin{array}{c}\text { Lower Thorax/ } \\
\text { upper Abdominal Cavity }\end{array}$ & Neck Area \\
\hline Number of lasers & 4 & 4 \\
\hline Wavelength (nm) & 905 & 905 \\
\hline Frequency $(\mathrm{Hz})$ & 250 & 250 \\
\hline Peak power $(\mathrm{W})$-each & 50 & 50 \\
\hline $\begin{array}{l}\text { Average mean optical output } \\
(\mathrm{mW}) \text {-each }\end{array}$ & 1.25 & 1.25 \\
\hline Power density $\left(\mathrm{mW} / \mathrm{cm}^{2}\right)$-each & 3.91 & 3.91 \\
\hline Energy density $\left(\mathrm{J} / \mathrm{cm}^{2}\right)$-each & 0.234 & 0.234 \\
\hline Dose $(\mathrm{J})$-each & 0.075 & 0.075 \\
\hline Spot size of laser $\left(\mathrm{cm}^{2}\right)$-each & 0.32 & 0.32 \\
\hline Number of red LEDs & 8 & 8 \\
\hline Wavelength of red LEDs (nm) & 633 & 633 \\
\hline Frequency $(\mathrm{Hz})$ & 2 & 2 \\
\hline Average optical output (mW)-each & 25 & 25 \\
\hline Power density $\left(\mathrm{mW} / \mathrm{cm}^{2}\right)$-each & 29.41 & 29.41 \\
\hline Energy density $\left(\mathrm{J} / \mathrm{cm}^{2}\right)$-each & 1.765 & 1.765 \\
\hline Dose $(\mathrm{J})$-each & 1.50 & 1.50 \\
\hline Spot size of red LED $\left(\mathrm{cm}^{2}\right)$-each & 0.85 & 0.85 \\
\hline Number of infrared LEDs & 8 & 8 \\
\hline Wavelength of infrared LEDs (nm) & 850 & 850 \\
\hline Frequency $(\mathrm{Hz})$ & 250 & 250 \\
\hline Average optical output (mW)-each & 40 & 40 \\
\hline Power density $\left(\mathrm{mW} / \mathrm{cm}^{2}\right)$-each & 71.23 & 71.23 \\
\hline Energy density $\left(\mathrm{J} / \mathrm{cm}^{2}\right)$-each & 4.286 & 4.286 \\
\hline Dose $(\mathrm{J})$-each & 2.40 & 2.40 \\
\hline Spot size of infrared LED $\left(\mathrm{cm}^{2}\right)$-each & 0.56 & 0.56 \\
\hline Magnetic field $(\mathrm{mT})$ & 110 & 110 \\
\hline Irradiation time per site (sec) & 60 & 60 \\
\hline Total dose per site $(\mathrm{J})$ & 31.50 & 31.50 \\
\hline
\end{tabular}


Table 1. Cont.

\begin{tabular}{|c|c|c|}
\hline & $\begin{array}{c}\text { Lower Thorax/ } \\
\text { upper Abdominal Cavity }\end{array}$ & Neck Area \\
\hline Number of irradiated sites & 6 & 1 (bilaterally) \\
\hline $\begin{array}{l}\text { Total dose delivered to the muscle } \\
\text { group }(\mathrm{J})\end{array}$ & 189.00 & 31.50 (bilaterally) \\
\hline Aperture of device $\left(\mathrm{cm}^{2}\right)$ & 33 & 33 \\
\hline Application mode & $\begin{array}{l}\text { Cluster probe held } \\
\text { stationary in skin contact } \\
\text { with a 90-degree angle } \\
\text { and slight pressure }\end{array}$ & $\begin{array}{l}\text { Cluster probe held } \\
\text { stationary in skin contact } \\
\text { with a 90-degree angle } \\
\text { and slight pressure }\end{array}$ \\
\hline
\end{tabular}

LED: light-emitting diode.

\subsection{Post-Treatment Outcomes}

After 10 days of PBMT-sMF treatment, the $\mathrm{SpO}_{2}$ of the patient increased from $89 \%$ to $93 \%$ at $2 \mathrm{~L} / \mathrm{min}$ oxygen. After 40 days of treatment with PBMT-sMF, the patient was able to leave the oxygen support. After 45 days, at the end of treatment with PBMT-sMF, the patient's $\mathrm{SpO}_{2}$ was at $96-98 \%$. Finally, in the last evaluation, 4 months after PBMT-sMF treatment, the patient's $\mathrm{SpO}_{2}$ was at $98 \%$ (Table 2).

Table 2. Post-treatment outcomes.

\begin{tabular}{cccccc}
\hline Variables. & Baseline & $\begin{array}{c}\text { Discharge from } \\
\text { Hospital }\end{array}$ & $\begin{array}{c}\text { 10 Days of } \\
\text { PBMT-sMF }\end{array}$ & $\begin{array}{c}\text { After End of } \\
\text { Treatment }\end{array}$ & $\begin{array}{c}\text { 4 Months } \\
\text { Follow-Up }\end{array}$ \\
\hline $\mathrm{SpO}_{2}(\%)$ & 74 & 89 & 93 & $96-98$ & 98 \\
$\mathrm{RALE}$ & 7 & - & 8 & - & 0 \\
\hline
\end{tabular}

$\mathrm{SpO}_{2}$ = peripheral oxygen saturation; RALE = radiographic assessment of lung edema; PBMT-sMF: photobiomodulation therapy combined with static magnetic field.

Figure 2 shows the extent of SARS-CoV-2 infection in the lungs. In addition, this was the basis for measuring the RALE score. When the patient was admitted to the hospital, his RALE score was 7 (Figure 2A). However, on the second chest radiograph, his RALE score worsened to 8 (Figure 2B). Finally, on the last radiograph, at 4 months follow-up evaluation, his RALE score was 0 , indicating total recovery of the lungs (Figure 2C) (Table 2).
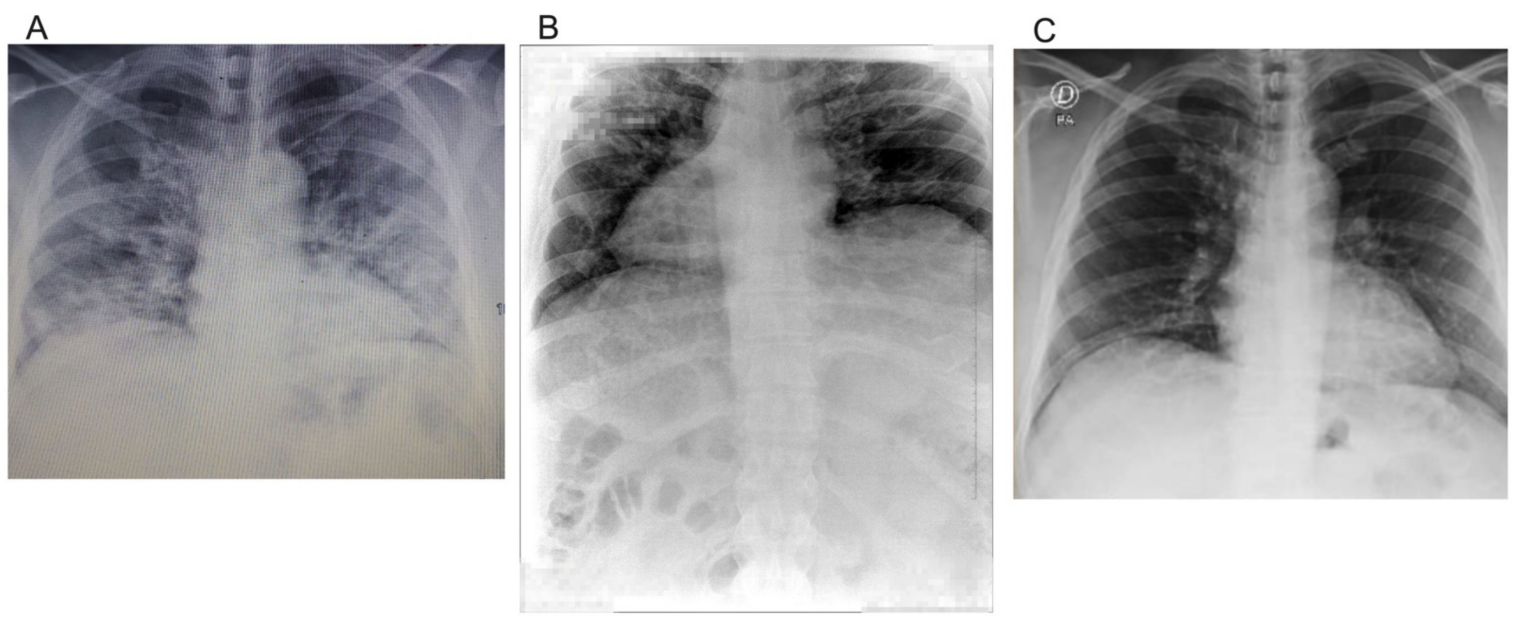

Figure 2. Radiological findings at baseline, 10 days after intervention and 4 months follow-up. Radiological findings at baseline (A). At baseline, the extension of consolidation or ground glass opacity was greater than $75 \%$ in the right lung, and $50-75 \%$ in the left lung. The figure is owned by the patient. Radiological findings at 10 days after starting the PBMT-sMF treatment (B). 10 days after starting the PBMT-sMF treatment, the extension of consolidation or ground glass opacity was greater than $75 \%$ in both lungs. The figure is owned by the patient. Radiological findings at 4 months follow-up evaluation (C). At 4 months follow-up evaluation, there was no consolidation or ground glass opacity in both lungs. The figure is owned by the patient. 
At the 4 months follow-up, spirometry was performed to assess the sequelae after COVID-19. Respiratory mechanics parameters were found according to their prediction, that is, what is normal in both the pre-and post-bronchodilator (Figure 3). The patient did not report any adverse events associated with the use of PBMT-SMF.

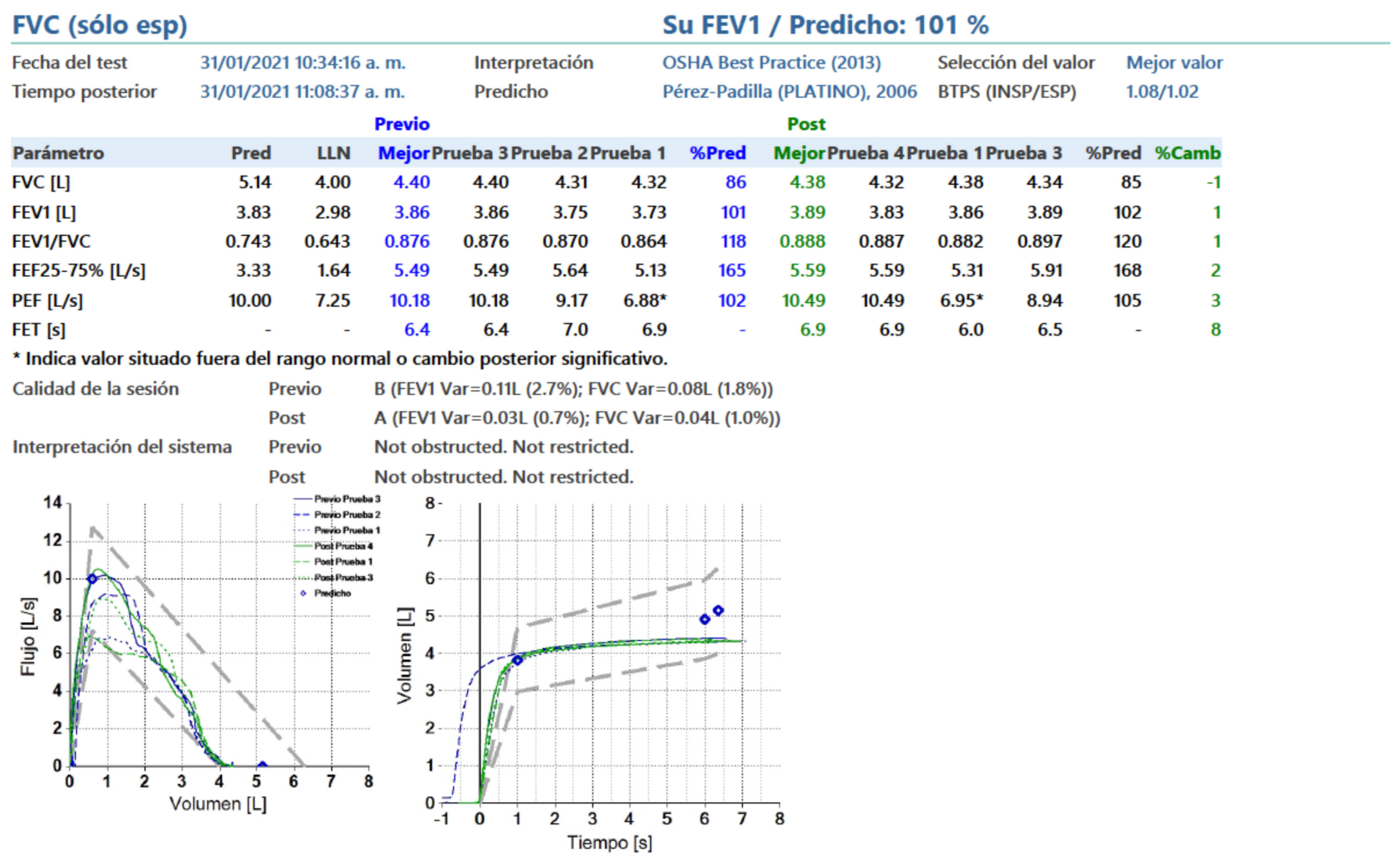

Figure 3. Spirometry-pulmonary function tests at 4 months follow-up evaluation. The respiratory mechanics parameters were found according to their prediction, that is, what is normal in both the pre-and post-bronchodilator. The figure is owned by the patient.

\section{Discussion}

This case report showed that the patient treated with PBMT-sMF for 45 days presented an increase in $\mathrm{SpO}_{2}$, in addition to improvements in oxygen support requirements, pulmonary severity scores, and radiological findings. In addition, during the 45-day treatment course with PBMT-sMF, the patient was able to leave the oxygen support. At follow-up 4 months after PBMT-sMF treatment, the clinical recovery of the patient was total, which was mainly proven by the pulmonary recovery.

These findings are consistent with the effects previously demonstrated by PBMT alone or by PBMT-sMF, such as the regeneration of tissues and modulation of the inflammatory process in several tissues [6,7] and in the respiratory system [8], as well as decreased pulmonary fibrosis and improvements in pulmonary functional capacity [11,21]. In addition, our findings corroborate a previous case report that demonstrated that PBMT treatment in a patient with severe COVID-19 was beneficial in reducing inflammatory markers and improving respiratory indices and radiological findings [16]. Moreover, another previous case report demonstrated improvements in respiratory indices, oxygen requirements, and radiological findings in a patient with severe COVID-19 treated with PBMT [17]. It is important to highlight that the aforementioned case reports only showed short-term improvements in patients, while our study demonstrated that patients treated with PBMT-sMF for 45 days also had better clinical findings at the long-term evaluation (4 months after the treatment).

Patients with COVID-19 often progress to acute respiratory distress syndrome, with an increased presence of cytokines such as interleukin (IL)- $1 \beta$ and IL- 6 . The findings observed in the present case report suggest that PBMT-sMF could modulate the inflammatory process, 
possibly decreasing the levels of these cytokines, as observed in previous experimental studies [22,23]. Another possible mechanism of action is that PBMT-sMF may contribute to the modulation of the immune system by acting on interferons [24]. In addition, PBMT alone or PBMT-sMF can reduce the production of reactive oxygen species and may lead to reduced oxidative stress in this specific condition $[8,25]$. Finally, PBMT-sMF may have been beneficial in preserving and improving the respiratory muscles of the patient. Therefore, our findings suggest that PBMT-sMF, when applied to the accessory muscle of respiration, helps improve the inflammatory process caused by COVID-19 by modulating inflammatory markers and the immune system, in addition to reducing oxidative stress. The combination of these positive effects of the use of PBMT-sMF contributed to the improvement in tissue oxygenation, pulmonary inflammation, and general clinical condition, which helped in the total recovery of the patient.

Although, currently there are no known adverse effects related to the use of PBMT-sMF, there are some contraindications regarding its use that should be carefully observed, such as irradiation in areas of infection, active carcinoma, and the pelvic and thoracoabdominal region in pregnant women.

One of the strengths of this case report was that we performed a medium-term followup of the patient to verify the evolution of the outcomes after the use of PBMT-sMF. In addition, we observed clinically important outcomes for the patient, such as peripheral oxygen saturation and pulmonary changes. However, one of our limitations is that our study was retrospective; thus, we do not have all the outcomes collected at all timepoints. In addition, the patient was diagnosed and treated at the critical time of the COVID-19 outbreak. Thus, the health system was overwhelmed, and access to the hospital and tests were limited. Therefore, the patient had to perform the second chest X-ray in a veterinary clinic, because he was a veterinarian and had access to veterinary facilities, and other laboratory tests could not be performed.

To corroborate our findings, randomized controlled trials with rigorous methodological quality and an adequate sample size are needed to investigate the effects of PBMT-sMF on pulmonary complications after COVID-19 in the short-, medium-, and long-term. In addition, studies on the effects of PBMT-sMF on patients with COVID-19 are needed.

\section{Conclusions}

The use of multi-wavelength PBMT-sMF may potentially lead to the safe treatment of and recovery from long-term pulmonary complications after COVID-19 infection, with regards to the structural and functional aspects.

Author Contributions: Conceptualization, S.S.T., D.S.J. and E.C.P.L.-J.; Methodology, D.S.J. and E.C.P.L.-J.; Formal Analysis, S.S.T., D.S.J. and E.C.P.L.-J.; Writing-Original Draft Preparation, S.S.T.; Writing-Review and Editing, D.S.J. and E.C.P.L.-J. All authors have read and agreed to the published version of the manuscript.

Funding: Multi-Radiance Medical (Solon-OH, USA) provided therapeutic devices for this study. Professor Ernesto Cesar Pinto Leal-Junior received grant \#310281/2017-2 from the Brazilian Council of Science and Technology Development (CNPq). CNPq had no role in this case report. MultiRadiance Medical had no role in data collection, analysis, and interpretation.

Institutional Review Board Statement: Not applicable.

Informed Consent Statement: Written informed consent has been obtained from the patient(s) to publish this paper.

Data Availability Statement: All datasets generated for this study are included in the article.

Conflicts of Interest: Ernesto Cesar Pinto Leal-Junior receives research support from Multi Radiance Medical (Solon-OH, USA), a laser device manufacturer. Shaiane Silva Tomazoni has a personal relationship with Ernesto Cesar Pinto Leal-Junior. Douglas Scott Johnson is an employee and shareholder of Multi Radiance Medical (Solon, OH, USA). 


\section{References}

1. World Health Organization (WHO). Report of the WHO-China Joint Mission on Coronavirus Disease 2019 (COVID-19). Available online: https:/ / www.who.int/docs / default-source/coronaviruse/who-china-joint-mission-on-covid-19-final-report.pdf (accessed on 17 February 2021).

2. Wiersinga, W.J.; Rhodes, A.; Cheng, A.C.; Peacock, S.J.; Prescott, H.C. Pathophysiology, Transmission, Diagnosis, and Treatment of Coronavirus Disease 2019 (COVID-19): A Review. JAMA 2020, 324, 782-793. [CrossRef]

3. Hoffmann, M.; Kleine-Weber, H.; Schroeder, S.; Krüger, N.; Herrler, T.; Erichsen, S.; Schiergens, T.S.; Herrler, G.; Wu, N.-H.; Nitsche, A.; et al. SARS-CoV-2 Cell entry depends on ACE2 and TMPRSS2 and is blocked by a clinically proven protease inhibitor. Cell 2020, 181, 271-280. [CrossRef]

4. Xu, Z.; Shi, L.; Wang, Y.; Zhang, J.; Huang, L.; Zhang, C.; Liu, S.; Zhao, P.; Liu, H.; Zhu, L.; et al. Pathological findings of COVID-19 associated with acute respiratory distress syndrome. Lancet Respir Med. 2020, 8, 420-422, Erratum in: Lancet Respir Med. 2020, 25. [CrossRef]

5. Huang, C.; Huang, L.; Wang, Y.; Li, X.; Ren, L.; Gu, X.; Kang, L.; Guo, L.; Liu, M.; Zhou, X.; et al. 6-month consequences of COVID-19 in patients discharged from hospital: A cohort study. Lancet 2021, 397, 220-232. [CrossRef]

6. Leal-Junior, E.C.P.; Lopes-Martins, R.Á.B.; Bjordal, J.M. Clinical and scientific recommendations for the use of photobiomodulation therapy in exercise performance enhancement and post-exercise recovery: Current evidence and future directions. Braz J. Phys. Ther 2019, 23, 71-75. [CrossRef] [PubMed]

7. Bjordal, J.M.; Johnson, M.I.; Iversen, V.; Aimbire, F.; Lopes-Martins, R.A. Low-level laser therapy in acute pain: A systematic review of possible mechanisms of action and clinical effects in randomized placebo-controlled trials. Photomed Laser Surg 2006, 24, 158-168. [CrossRef]

8. De Lima, F.M.; Vitoretti, L.; Coelho, F.; Albertini, R.; Breithaupt-Faloppa, A.C.; de Lima, W.T.; Aimbire, F. Suppressive effect of low-level laser therapy on tracheal hyperresponsiveness and lung inflammation in rat subjected to intestinal ischemia and reperfusion. Lasers Med. Sci 2013, 28, 551-564. [CrossRef]

9. Aimbire, F.; Bjordal, J.M.; Iversen, V.V.; Albertini, R.; Frigo, L.; Pacheco, M.T.; Castro-Faria-Neto, H.C.; Chavantes, M.C.; Labat, R.M.; Lopes-Martins, R.A. Low level laser therapy partially restores trachea muscle relaxation response in rats with tumor necrosis factor alpha-mediated smooth airway muscle dysfunction. Lasers Surg Med. 2006, 38, 773-778. [CrossRef]

10. Silva, V.R.; Marcondes, P.; Silva, M.; Villaverde, A.B.; Castro-Faria-Neto, H.C.; Vieira, R.P.; Aimbire, F.; de Oliveira, A.P. Low-level laser therapy inhibits bronchoconstriction, Th2 inflammation and airway remodeling in allergic asthma. Respir Physiol Neurobiol 2014, 194, 37-48. [CrossRef]

11. De Souza, G.H.M.; Ferraresi, C.; Moreno, M.A.; Pessoa, B.V.; Damiani, A.P.M.; Filho, V.G.; Dos Santos, G.V.; Zamunér, A.R. Acute effects of photobiomodulation therapy applied to respiratory muscles of chronic obstructive pulmonary disease patients: A double-blind, randomized, placebo-controlled crossover trial. Lasers Med. Sci 2020, 35, 1055-1063. [CrossRef]

12. Friedmann, H.; Lipovsky, A.; Nitzan, Y.; Lubart, R. Combined magnetic and pulsed laser fields produce synergistic acceleration of cellular electron transfer. Laser Ther 2009, 18, 137-141. [CrossRef]

13. Vanin, A.A.; Verhagen, E.; Barboza, S.D.; Costa, L.O.P.; Leal-Junior, E.C.P. Photobiomodulation therapy for the improvement of muscular performance and reduction of muscular fatigue associated with exercise in healthy people: A systematic review and meta-analysis. Lasers Med. Sci 2018, 33, 181-214. [CrossRef] [PubMed]

14. Langella, L.G.; Casalechi, H.L.; Tomazoni, S.S.; Johnson, D.S.; Albertini, R.; Pallotta, R.C.; Marcos, R.L.; de Carvalho, P.T.C.; Leal-Junior, E.C.P. Photobiomodulation therapy (PBMT) on acute pain and inflammation in patients who underwent total hip arthroplasty-a randomized, triple-blind, placebo-controlled clinical trial. Lasers Med. Sci 2018, 33, 1933-1940. [CrossRef]

15. Tomazoni, S.S.; Costa, L.O.P.; Joensen, J.; Stausholm, M.B.; Naterstad, I.F.; Ernberg, M.; Leal-Junior, E.C.P.; Bjordal, J.M. Photobiomodulation therapy is able to modulate $\mathrm{PGE}_{2}$ levels in patients with chronic non-specific low back pain: A randomized placebo-controlled trial. Lasers Surg Med. 2021, 53, 236-244. [CrossRef]

16. Sigman, S.A.; Mokmeli, S.; Vetrici, M.A. Adjunct low level laser therapy (LLLT) in a morbidly obese patient with severe COVID-19 pneumonia: A case report. Can. J. Respir Ther 2020, 56, 52-56. [CrossRef] [PubMed]

17. Sigman, S.A.; Mokmeli, S.; Monici, M.; Vetrici, M.A. A 57-Year-Old African American Man with Severe COVID-19 Pneumonia Who Responded to Supportive Photobiomodulation Therapy (PBMT): First Use of PBMT in COVID-19. Am. J. Case Rep. 2020, 21, e926779. [CrossRef] [PubMed]

18. Warren, M.A.; Zhao, Z.; Koyama, T.; Bastarache, J.A.; Shaver, C.M.; Semler, M.W.; Rice, T.W.; Matthay, M.A.; Calfee, C.S.; Ware, L.B. Severity scoring of lung oedema on the chest radiograph is associated with clinical outcomes in ARDS. Thorax 2018, 73, 840-846. [CrossRef] [PubMed]

19. Wong, H.Y.F.; Lam, H.Y.S.; Fong, A.H.; Leung, S.T.; Chin, T.W.; Lo, C.S.Y.; Lui, M.M.; Lee, J.C.Y.; Chiu, K.W.; Chung, T.W.; et al. Frequency and Distribution of Chest Radiographic Findings in Patients Positive for COVID-19. Radiology 2020, 296, E72-E78. [CrossRef]

20. De Marchi, T.; Frâncio, F.; Ferlito, J.V.; Weigert, R.; de Oliveira, C.; Merlo, A.P.; Pandini, D.L.; Pasqual-Júnior, B.A.; Giovanella, D.; Tomazoni, S.; et al. Effects of Photobiomodulation Therapy Combined with Static Magnetic Field in Severe COVID-19 Patients Requiring Intubation: A Pragmatic Randomized Placebo-Controlled Trial. J. Inflamm Res. 2021, 14, 3569-3585. [CrossRef] 
21. De Brito, A.A.; da Silveira, E.C.; Rigonato-Oliveira, N.C.; Soares, S.S.; Brandao-Rangel, M.A.R.; Soares, C.R.; Santos, T.G.; Alves, C.E.; Herculano, K.Z.; Vieira, R.P.; et al. Low-level laser therapy attenuates lung inflammation and airway remodeling in a murine model of idiopathic pulmonary fibrosis: Relevance to cytokines secretion from lung structural cells. J. Photochem Photobiol B 2020, 203, 111731.

22. Oliveira, M.C., Jr.; Greiffo, F.R.; Rigonato-Oliveira, N.C.; Custódio, R.W.A.; Silva, V.R.; Damaceno-Rodrigues, N.R.; Almeida, F.M.; Albertini, R.; Lopes-Martins, R.Á.B.; de Oliveira, L.V.F.; et al. Low level laser therapy reduces acute lung inflammation in a model of pulmonary and extrapulmonary LPS-induced ARDS. J. Photochem Photobiol B 2014, 134, 57-63. [CrossRef] [PubMed]

23. Cury, V.; de Lima, T.M.; Prado, C.M.; Pinheiro, N.; Ariga, S.K.; Barbeiro, D.F.; Moretti, A.I.; Souza, H.P. Low level laser therapy reduces acute lung inflammation without impairing lung function. J. Biophotonics 2016, 9, 1199-1207. [CrossRef] [PubMed]

24. Huang, T.J.; MacAry, P.A.; Wilke, T.; Kemeny, D.M.; Chung, K.F. Inhibitory effects of endogenous and exogenous interferongamma on bronchial hyperresponsiveness, allergic inflammation and T-helper 2 cytokines in Brown-Norway rats. Immunology 1999, 98, 280-288. [CrossRef]

25. Tomazoni, S.S.; Machado, C.D.S.M.; De Marchi, T.; Casalechi, H.L.; Bjordal, J.M.; de Carvalho, P.T.C.; Leal-Junior, E.C.P. Infrared low-level laser therapy (photobiomodulation therapy) before intense progressive running test of high-level soccer players: Effects on functional, muscle damage, inflammatory, and oxidative stress markers- a randomized controlled trial. Oxid Med. Cell Longev 2019, 6239058. [CrossRef] 